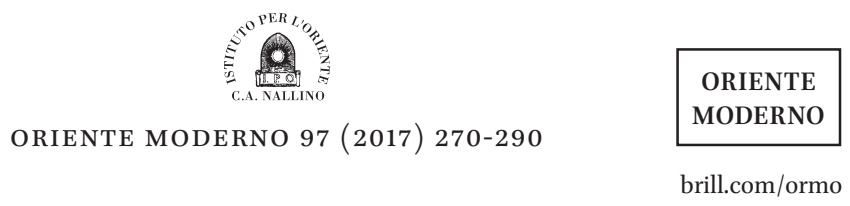

\title{
L'economia islamica: alternativa apparente o reale?
}

\author{
Valentino Cattelan \\ Saudi-Spanish Center for Islamic Economics and Finance-IE Business School, \\ Madrid \\ cattelanvalentino@libero.it
}

\begin{abstract}
This article deals with the nature of Islamic economics as a scientific paradigm which claims to be alternative to conventional economic thinking.

To critically evaluate this claim, the work investigates the peculiar religious and moral principles that shape the idea of social justice in Islam. Subsequently, it outlines how Islamic economics derives from these principles a specific conceptualization of property rights and commercial relations that embraces parameters of (1) primacy of real economy; (2) transactional equilibrium; (3) and profit- and risk-sharing.

By endorsing the conceptual autonomy of Islamic economics from conventional capitalism, the article also refers to the current emergence of the Islamic financial market at a global stage, and the possible implications for a plural financial system in the future.
\end{abstract}

\section{Keywords}

Islamic economics - Islamic finance - paradigm - social justice - property rights capitalism.

* Questo scritto trae il proprio spunto originario da alcune riflessioni formulate in seno alla conferenza Le primavere arabe. Prospettive euro-mediterranee, svoltasi nel 2012 presso l'Università degli Studi di Catania, ed organizzata dalla Prof.ssa Laura Bottini; riflessioni successivamente sviluppate in una serie di scritti pubblicati dall'Autore in lingua inglese, richiamati nel corso del testo e in questa sede organicamente rielaborati. Desidero pertanto ringraziare sia la Prof.ssa Bottini, che gli altri relatori del panel Economia, lavoro e società (Prof.ssa Biancamaria Scarcia Amoretti, in veste di moderatrice dei lavori; Prof. Youssef Courbage; Prof.ssa Cristiana Baldazzi) per la discussione e gli utili commenti ricevuti già a Catania nel 2012, da cui le sopra citate pubblicazioni hanno spesso tratto beneficio intellettuale. 
A partire da quelle che gli osservatori internazionali avevano generosamente descritto come "Primavere", l'evoluzione politico-sociale del mondo musulmano ha contraddistinto il dibattito euro-mediterraneo negli ultimi anni, fino a trasformarsi in questione di interesse domestico per l'Europa in relazione ai flussi migratori dal Nord Africa e Vicino Oriente, come effetto conseguente dell'espansione militare dell'auto-proclamatosi "Califfato". Tuttavia, come il sostegno dell'Occidente parve esitante verso l'inatteso slancio democratico dei popoli arabi, per la persistenza di questioni di sicurezza geo-politica (Roy 2012), così la comunità internazionale tarda tutt'oggi a contrapporre all'avanzata del Califfato una strategia di contenimento capace tanto di tutelare la popolazione locale avverso gli attacchi terroristici, quanto di sostenere forze militari nazionali contro la guerra in atto. Come risultato, se da un lato le sfide poste dalle Primavere arabe, sia di matrice geo-politica che economicosociale (Malik - Awadallah, 2011), non seppero trovare adeguate risposta in sede euro-mediterranea, così oggi la gestione dei flussi migratori verso l'Europa, drammatico effetto della minaccia del "Califfato nero", rimangono in balìa di riflussi nazionalistici e ragioni di opportunità politica dei singoli stati dell'Unione europea.

Non è obiettivo di questo scritto indagare le ragioni dell'insuccesso delle Primavere arabe od esaminare le cause politiche della nascita e dell'espansione del Califfato, quanto rilevare come sia nell'alveo delle prime, per quanto marginalmente (e soprattutto in relazione all'ascesa al potere dei Fratelli Musulmani in Egitto), così nella propaganda mediatica del secondo, siano emersi richiami all'"economia islamica" come strategia di politica economica alternativa al capitalismo occidentale, alla cui comprensione questo lavoro è appunto rivolto.

Successivamente alle rivolte civili, infatti, esponenti del nuovo governo egiziano dei Fratelli Musulmani (Lofty, 2012; Lofty; Vizcaino, 2012), così come di quello tunisino di Harakat al-Nahdah, il Movimento della Rinascita (Vizcaino, 2012), avevano dato voce alla volontà di promuovere a livello nazionale un'economia e una finanza di matrice musulmana, ossia volte ad applicare i principi dell'Islam nella gestione dell'economia reale, del credito e del risparmio. Intenti rientrati dopo la caduta del governo di Muhammad Mursī in Egitto nel 2013, anche se apparentemente non del tutto abbandonati (per ragioni di convenienza economico-diplomatica nei rapporti coi regimi del Golfo) da parte del governo neo-liberale di 'Abd al-Fattāḥ al-Sīsī, come appare dal resoconto della recente conferenza di Sharm al-Shaykh (13-15 marzo 2015: Solovieva - Wahba, 2015). Allo stesso modo, anche la Tunisia post-rivoluzionaria ha considerato, e continua a considerare, il canale dell'economia islamica come segmento della propria strategia di attrazione di capitali šarīah-compliant dal Golfo e 
dal Sud-Est asiatico (AFM I, 2015), ossia dalle regioni dove si è avuto negli ultimi decenni il massimo sviluppo della c.d. "finanza islamica", che dell'applicazione dei principi sciaraitici in ambito commerciale costituisce il (ricco) sbocco operativo (al riguardo, si veda oltre in questo paragrafo).

Relativamente al Califfato, mentre nessuno studio scientifico si è ancora occupato del modello economico di riferimento del nuovo "Stato" islamico, il "marketing della propaganda" che ne caratterizza l'espansione, sia mediatica sia geo-politica (in tal senso, Ballardini, 2015), si è spinto a proclamare tra i propri obiettivi quello di "spazzare via il sistema finanziario capitalistico di schiavitù, sostenuto da un pezzo di carta chiamato dollaro", come recentemente riportato anche dai mass-media italiani (Serafini, 2015). Si auspica a tal fine la reintroduzione del dīnār d'oro e del dirham d'argento come monete ufficiali dello "Stato", richiamandosi direttamente alla dottrina del Profeta. Ebbene, se da un lato questo progetto di politica monetaria risente di scarsa considerazione storica della realtà socio-economica dei tempi del Profeta, certamente non riproponibile nell'età contemporanea e, checché ne dica la propaganda del Califfato, non affatto basata sulla esclusiva circolazione del dinaro d'oro come mezzo di scambio (Brunschvig; 1967, Ray, 1997; Udovitch, 1970), dall'altro lato ripropone, come detto, il riferimento ad un'istanza economica islamica i cui fondamenti etici, religiosi e razionali-programmatici è opportuno approfondire in questa sede, sia in un'ottica comparativa con il capitalismo "convenzionale" di stampo occidentale, sia per offrire degli spunti di riflessione rispetto ad un'attualità del mondo musulmano dove, per ragioni di opportunità, di identità politica o di propaganda, a tale dottrina si fa sempre più spesso riferimento.

Che cosa si intende, allora, con la locuzione "economia islamica"? In uno dei pochi articoli disponibili in materia nelle riviste economiche di lingua italiana si evidenzia come

il Corano è considerato dall'Islam e da tutti i musulmani come espressione degli effettivi pronunciamenti di Dio. Di conseguenza i valori economici basilari da esso indicati sono invariabili, eterni, indiscutibili ... Un'economia può essere classificata come islamica solo se sceglie di fondare le sue politiche economiche e pubbliche su tali dottrine essenziali.

ASKARI; TAGHAVI, 2005, 175-176

Se fino a poco tempo fa l'argomento "economia islamica" costituiva un "fortino" con bastioni sorretti da pochi studi specialistici in lingua italiana (Chapra, 1979; Ismail, 1980; Scarcia Amoretti, 1986 e 1988; AA.VV., 1988), ma con mura rafforzate dall'interesse di alcuni giuristi verso il modello della banca islamica 
(Piccinelli, 1996), le peculiarità della "via islamica" all'economia hanno di recente ottenuto ben più ampia indagine.

Tale rinnovato interesse, a ben vedere, non è tanto da collegare all'attualità politica del mondo musulmano del Nord Africa e del Vicino Oriente, quanto alla recente globalizzazione del mercato della c.d. "finanza islamica", che a partire dagli anni Settanta del secolo scorso ha cercato di rendere operativi i meccanismi dell'economia šarĩah-compliant, attraverso prodotti in linea con le prescrizioni coraniche. Prodotti, in particolare, strutturati (da un punto di vista formalistico-legale) secondo la proibizione della remunerazione dei capitali ad interesse $(r i b \bar{a})$, della incertezza dell'oggetto contrattuale (jarar) e di ogni alea e speculazione (maysir), cui si affiancano il divieto di investimenti in armi, alcol, industria del gioco e carne suina; nonché diretti al contrario alla promozione (da un punto di vista economico-sostanziale) di progetti finanziari di impatto sociale attraverso forme di microcredito, finanza di progetto o tramite la riscoperta del waqf come istituto della tradizione giuridica musulmana "modernizzato" allo scopo di una gestione funzionale di patrimoni a sostegno dello sviluppo locale.

In questo modo, soprattutto nelle ultime due decadi, il mercato della finanza islamica si è notevolmente rafforzato, crescendo annualmente con tassi compresi tra il 10 ed il 15 per cento sia in ambito bancario che assicurativo (industria del c.d. takāful, assicurazione tramite "reciproca garanzia") e tramite la diffusione di nuovi strumenti finanziari (c.d. șukūk, volgarmente conosciuti come "bond islamici", pur avendo ben poco a che fare con le obbligazioni tradizionali, e dovendo essere più correttamente descritti come titoli di partecipazione condivisa in un investimento comune).

Pur essendo difficile stimare le dimensioni operative della finanza islamica, che ad oggi coprirebbe comunque non più del 2-3 per cento delle transazioni mondiali, il mercato šarîah-compliant si è ormai diffuso a livello globale, dal Golfo (Arabia Saudita, Qatar, EAU), Iran e Sud-Est asiatico (rimanendo la Malaysia il mercato dei capitali più avanzato nella regione), all'Europa (TheBanker, 2013) e soprattutto nel Regno Unito (Cattelan, 2013a). Contemporaneamente, anche la letteratura scientifica in materia si è ampiamente estesa e comprende ormai una quantità innumerevole di pubblicazioni, sia di tipo introduttivo (Ayub, 2007), che di impostazione politico-economica (Warde, 2000; Tripp, 2006), economico-giuridica (Vogel - Hayes, 1998); oppure di impianto critico (El-Gamal, 2006); o più concentrata verso la modernizzazione del diritto islamico nel contesto finanziario attuale (Ercanbrack, 2015).

Prendendo atto dello sviluppo di un mercato finanziario islamico a livello globale (e complice più di recente la crisi finanziaria europea, che ha spinto 
verso la ricerca di attrazione di liquidità da nuovi mercati), anche numerosi economisti italiani hanno diretto la loro attenzione al capitalismo šarìahcompliant (Aliboni, 2003; Vadalà, 2004; Giustiniani, 2006; Hamaui; Mauri, 2009; Dell'Atti; Miglietta, 2009; Porzio, 2009; Khan; Porzio, 2010; Biancone, 2012), con un corrispondente fiorire di convegni e seminari a tema. Al riguardo, una giornata di studio sulla finanza islamica è stata ad esempio organizzata dalla Banca d'Italia nel 2009, con una successiva pubblicazione in materia (Gomel, 2010); anche la соNSOB, Commissione Nazionale per la Società e la Borsa, ha dedicato uno studio alle prospettive di integrazione della finanza islamica nel contesto giuridico ed economico italiano (Alvaro, 2014); dal 2014, tra l'altro, si svolge a Torino il Turin Islamic Economic Forum, volto appunto alla divulgazione degli aspetti dell'economia islamica di maggior interesse per gli investitori internazionali ed italiani. Nonostante tali iniziative, tuttavia, l'economia islamica non è ancora oggi oggetto specifico di studio (anche a livello avanzato, di master o dottorato) nei corsi delle facoltà italiane di economia, tranne rarissime eccezioni; più spesso, invece, lezioni di economia islamica vengono collocate nei curricula di corsi di cooperazione allo sviluppo o studi internazionali e di matrice politico-sociale (Francesca, 2013 Atzori, 2010); nuovi titoli sono stati pubblicati inoltre in chiave divulgativa (Guandalini - Uckmar, 2015). Per quanto riguarda le facoltà di giurisprudenza, in aumento sono le opere dei giuristi italiani che si spingono oltre le mura del "fortino" dell'economia islamica (Piccinelli, 1996; Gimigliano - Rotondo, 2006; Russo, 2014), con corrispondenti seminari specifici sul diritto della finanza islamica ed il diritto contrattuale e commerciale musulmano.

In linea con questo crescente interesse di stampo multi-disciplinare ed alla luce, come detto, di un'attualità in cui la via islamica al capitalismo emerge sempre più di frequente nel dibattito politico, questo breve contributo vuole offrire alcuni spunti riflessivi sull'economia islamica, presentandone in chiave critica i presupposti logico-morali, i principali contenuti e le sue finalità essenziali, e valutando in particolare se essa offra o meno una reale alternativa al modello capitalista occidentale, o se questa alternativa sia solo apparente e meramente riconducibile a spinte di matrice politico-identitaria.

A tal riguardo la riflessione sarà incentrata sul rapporto tra fede, etica e razionalità nell'economia islamica $(\S 2)$, mostrando come, se una sua specifica autonomia semantica sussiste, questa possa essere individuata nella ricerca di modalità alternative di gestione dell'economia reale, del credito e del rischio, sullo sfondo di una diversa concettualizzazione del bene economico e dei diritti patrimoniali del singolo $\left(\S_{3}\right)$. Infine, alcune considerazioni conclusive riguarderanno l'apporto che l'economia islamica può offrire in termini di pluralismo rispetto alla teoria classica del modello capitalista $(§ 4)$. 

sociale dell'Islam

Come indicato, l'economia islamica ha per esplicito obiettivo la proposizione di un modello di sviluppo economico fondato sui principi valoriali dell'Islam.

La letteratura in materia è cresciuta esponenzialmente tra gli anni Cinquanta e Ottanta del secolo scorso, soprattutto con opere di autori di fede musulmana (Rodinson, 1966; Udovitch, 1970). Se il numero complessivo di pubblicazioni poteva essere calcolato alla fine degli anni Ottanta in circa 680 titoli, di cui il 30\% in arabo, il 33\% in inglese e il $27 \%$ in urdu (Philipp, 1990:117), nelle ultime due decadi, soprattutto con il crescere del mercato finanziario islamico (che, come vedremo meglio in seguito, si contraddistingue per operazioni interest-free e asset-backed: Vogel - Hayes, 1998), la letteratura si è esponenzialmente moltiplicata, fino a rendere impossibile stilarne una bibliografia completa, se non per opere chiave di riferimento (Islahi, 2007). Contemporaneamente i centri di studio sull'economia islamica si sono diffusi a livello globale: a titolo meramente esemplificativo possono essere ricordati, in Vicino Oriente, l'Islamic Economics Institute dell'Università King Abdul Aziz di Ğeddah (Arabia Saudita); in Asia, l'International Islamic University of Malaysia (Kuala Lumpur); in Europa, il Durham Centre for Islamic Economics and Finance (UK) e il Saudi-Spanish Center for Islamic Economics and Finance dell'Instituto de Empresa di Madrid (Spagna).

Ma quali sono le tematiche oggetto della letteratura economica islamica?

In estrema sintesi, possono essere identificati alcuni argomenti-chiave. Soprattutto fino agli anni Ottanta numerosi contributi riguardavano, da un lato, la critica al comunismo ed al socialismo, sposando l'Islam, come vedremo in seguito, una filosofia economica di stampo capitalistico; dall'altro, il diniego dell'interesse (identificato in toto nell'usura, indipendentemente dal tasso applicato) come modalità di remunerazione del capitale, sulla base del celeberrimo divieto di ribā sancito in Corano II:275:

Coloro che praticano l'usura, il dì della Resurrezione sorgeran dai sepolcri come chi è reso epilettico dal contatto di Satana. Questo perché essi hanno detto: 'La compravendita è come l'usura'. Ma Dio ha permesso la compravendita [al-baý: da leggersi come attività di commercio in generale] e proibito l'usura $[r i b \bar{a}]$.

Caduto il muro di Berlino e con esso il valore politico della critica dogmatica al modello economico comunista, la letteratura islamica si è successivamente focalizzata sulla concettualizzazione di un sistema alternativo di scambi 
commerciali capaci di promuovere la giustizia sociale, l'equità e la responsabilità individuale attraverso l'applicazione dei precetti dell'Islam, al fine del benessere terreno e spirituale dell'uomo ( falāh $)$, ottenuto appunto attraverso la rimozione dell'usura e di ogni forma di interesse sul capitale $(r i b \bar{a})$; l'eliminazione della speculazione ('garar o maysir) dai mercati finanziari; nonché attraverso la promozione di uno Stato sociale che si faccia promotore della raccolta e distribuzione dell'elemosina obbligatoria (zakāh).

La prosperità economica che l'adesione ai valori dell'Islam potrebbe garantire (o meglio garantisce tout court, per il credente musulmano) viene coniugata in questa letteratura nei termini di una giustizia ('adl) e di un diritto oggettivo e soggettivo (haqq) offerti dalla "Via rivelata" (šarīah), come obiettivi (maqāșid) della volontà divina. Così Al-Makarim (1974:35) definisce l'economia islamica come "la scienza che ha per oggetto il benessere e la sua relazione con l'essere umano dal punto di vista della realizzazione della giustizia in ogni forma di attività economica". Hasanuzzaman (1984:52) ne parla in termini di "conoscenza ed applicazione delle ingiunzioni e dei precetti della šarīah che prevengono ogni ingiustizia nell'acquisizione e disposizione delle risorse materiali, in modo da offrire soddisfacimento all'uomo e renderlo in grado di adempiere ai suoi obblighi verso Iddio e la società". In linea con questo approccio, Khan (1984:55) individua l'obiettivo essenziale dell'economia islamica nello studio della "prosperità ( falăḥ) dell'uomo per mezzo dell'organizzazione delle risorse in base a criteri di cooperazione e partecipazione" (su tali criteri torneremo tra poco); mentre Chapra (2000:125) si riferisce alla "realizzazione del benessere umano attraverso l'allocazione e distribuzione delle risorse ... in conformità agli obiettivi (maqāṣid)" della šarīah.

Ai fini di questo saggio è importante sottolineare come tali presupposti valoriali conducano gli assertori dell'economia islamica non solo ad evidenziare i limiti del capitalismo occidentale (di cui si denuncia il materialismo, il consumismo e addirittura la mancanza di presupposti etici tout court), ma anche a rivendicare un'autonomia scientifica dell'economia islamica come paradigma alternativo a quello della letteratura economica convenzionale (senza spesso distinguere tra teoria economica classica, approccio istituzionale, approccio comportamentale, teoria dei beni comuni ... e così appiattando sfortunatamente la letteratura occidentale in un unicum da rigettare).

Ma entro quali limiti l'alternativa "professata" dagli economisti islamici può essere riconosciuta? E, a fortiori, possono asserzioni valoriali di matrice etico-religiosa fondare logicamente la pretesa di un paradigma scientifico alternativo (Kuhn, 1970)?

Si tratta evidentemente di quesiti complementari, dove questioni epistemologiche si legano a parametri etico-religiosi nella dimensione della giustizia 
economica. Tale pretesa di autonomia dogmatica è stata oggetto di numerosi rilievi critici, se non di giudizio esplicitamente negativo (Kuran, 1986), fino a essere bollata come ideologica ed espressione più di ragioni identitarie che di autentica prassi scientifica:

it seems unlikely that this discussion of Islamic economics will provide a genuine - and specifically Islamic at that — alternative to existing economic systems and theoretical approaches. But even the mere discussion can provide a sense of cultural identity and independence and may legitimize any number of specific economic measures a government may adopt. This makes it attractive to the Muslim intellectual and advantageous to any government that draws its legitimacy from Islam.

PHILIPP, 1990:139

L'utilità ideologica che l'economia islamica può avere per un governo che derivi la proprio legittimità dall'Islam richiama alla nostra attenzione proprio gli annunci dei governi egiziano e tunisino prima riportati in direzione dell'attrazione di capitali dal Golfo, che potrebbero essere allora facilmente giudicati in termini di pura opportunità politica, se non di cieca propaganda (come nel caso delle rivendicazioni del "Califfato nero").

Ma questo (pre-)giudizio sulla sostanziale a-scientificità dell'economia islamica pare a me eccessivamente aprioristico (e quindi altrettanto ideologico), soprattutto in relazione ad una genuina consapevolezza della complessità delle questioni coinvolte, nonché dello sviluppo ormai pluri-decennale della finanza islamica come segmento di stampo etico, alternativo alla finanza convenzionale.

In particolare, è necessaria a mio avviso una più approfondita riflessione preliminare sui rapporti tra religione ed etica nella definizione di una certa idea di "giustizia economica", prima di valutare se questa concettualizzazione etico-religiosa di giustizia incida o meno sulla sua successiva elaborazione scientifica.

Al riguardo, per esempio, è indubitabile che il fondamento religioso dell'economia islamica conduca ad una nozione di bene sociale che non si coniuga esclusivamente nei termini secolari dell'efficienza (come spesso accade nell'economia convenzionale di matrice occidentale), ma si declina anche nell'orizzonte di una dimensione escatologica di salvezza. Tale incidenza del fattore religioso sul dato economico, a ben vedere, non può essere comunque intesa in termini di assoluta eccezionalità: è noto, infatti, come anche nel capitalismo convenzionale possa essere individuato uno "spirito" (Weber), così come la teologia cristiana medievale ha certamente contribuito alla "redenzione" 
dell'usuraio nelle vesti del banchiere (Le Goff, 1986 e 2010), gettando così i semi per il capitalismo moderno (per l'apporto "conclusivo" dato dal calvinismo alla legittimazione dell'usura, si veda l'illuminante Nelson, 1949).

In definitiva, se la cultura secolare dell'Occidente ha adombrato le radici etico-religiose del proprio modello di crescita economica, ciò non significa affatto che tali radici siano state estirpate ex tunc. Nell'economia islamica, viceversa, radici diverse non solo esistono, ma vengono tutt'oggi culturalmente valorizzate sia come parametro di giustizia sociale che in termini di elaborazione scientifica.

Inoltre, parlando di economia islamica, si impongono evidentemente i contorni dogmatici dell'etica musulmana (Gimaret, 1980), con tutte le sue peculiarità. In altri termini, se un fondamento morale, come quello religioso, di certo sussiste nell'economia islamica (come esiste alle radici dell'economia convenzionale - cui spesso gli occhi occidentali non guardano con attenzione), tale etica va appunto culturalmente ascritta all'Islam, nella misura in cui, per esempio, la rimozione dell'interesse pecuniario rispondente al divieto di ribā (certamente "etico" per il musulmano credente) non per questo soddisfa immediati criteri morali per il cristiano di oggi (per il quale l'interesse, oggi, è pienamente "giusto"; Nelson, 1949).

In tale direzione, i risultati di una certa operazione economica vanno quindi di volta in volta giudicati secondo una "bilancia" ad hoc di giustizia sociale, che ne valuterà i modi secondo propri criteri etici: così, sia l'equilibrio finale della bilancia estesa a livello dell'intera comunità sociale (in termini di giustizia distributiva) che il rapporto tra i singoli bracci della bilancia (in termini di giustizia commutativa tra individui) viene eticamente modulato in modo diverso da culture/religioni diverse.

Guardando all'economia islamica, per esempio, ad essere primariamente strumento di giustizia distributiva non è solo la zakāt (l'elemosina rituale come uno dei pilastri dell'Islam), ma un impianto teorico di partecipazione/ cooperazione nella gestione del bene economico (sul punto, vedi in seguito). Il divieto di $r i b \bar{a}$, come quelli di jarar and maysir, sono invece mezzi di giustizia commutativa, laddove, al contrario, la percezione dell'interesse pecuniario (sulla base del riconoscimento dell'assunzione di un rischio di credito per la controparte, ad esempio, una banca) è percepito come legittimo nel capitalismo convenzionale. Al tempo stesso, se per il musulmano l'adempimento della volontà di Allāh ha in sé valore etico (in termini di giustizia legale, così come intesa nella teologia ortodossa ash'arita: Gimaret, 1990), il cristiano è abituato a valutare invece l'eticità dell'azione in relazione alla sostanza dei risultati piuttosto che alla forma dei mezzi. Per esemplificare, mentre per un musulmano 
la forma "società di capitali", mušārakah (con l'evidente esclusione di attività harām, come la commercializzazione di vino) è per se etica, in quanto aderente ai divieti di ribā, garar e maysir e capace di promuovere una condivisione del rischio di impresa (nonché per essere codificata da secoli nella letteratura del figh), per il cristiano il giudizio etico non si soffermerebbe sulla forma, guardando anche alla sostanza degli investimenti della società in esame (ad es., se si occupa di rimboschimento o della produzione di auto; se ha un impatto sociale o produce semplicemente profitto per gli azionisti; e via dicendo).

Non è possibile approfondire in questa sede questioni connesse alla concettualizzazione della giustizia economica in culture diverse (Cattelan, 2010). Tuttavia, questa riflessione preliminare sul rapporto tra religione ed etica nell'economia islamica è funzionale a comprendere se ed in quale misura le sue "asserzioni di valore" legittimino o meno la pretesa di un "autonomo paradigma in sede scientifica" (Cattelan, 2013c). A tal riguardo, l'immagine della "bilancia" (mizzān) come metafora di giustizia socio-economica (in ottica legale, commutativa e distributiva) si rivelerà utile a chiarire ulteriormente le differenze tra il pensiero economico occidentale e musulmano, fino a poter individuare un'eventuale autonomia concettuale del secondo rispetto al primo, sia relativamente all'idea di bene economico che alla natura dei diritti patrimoniali imputabili al singolo.

Dalla volontà divina alla natura dei diritti patrimoniali nell'Islam: le radici concettuali di un paradigma economico alternativo

Come precedentemente indicato, l'economia islamica sposa un modello di stampo capitalistico (non certo comunista o socialista), in cui lavoro e investimento sono gli strumenti legittimi per acquisire diritti di proprietà: l'accumulazione di ricchezza o il perseguimento del profitto attraverso forme di "accrescimento illecito" (riba $\bar{a}$ ), come l'interesse, o basate su elementi aleatori (garar) o speculativi (maysir), o sulla tesaurizzazione improduttiva, vengono viceversa rigettati.

Ebbene, tali principi etici (nel senso precedentemente ricondotto all'Islam) si nutrono di un'antropologia giuridico-economica che incide profondamente sulla costruzione della "bilancia" (mizzān) della giustizia sociale nell'economia islamica, fino a plasmare la definizione del suo paradigma scientifico, come cercherò di chiarire in questo paragrafo.

Nell'Islam, infatti, qualunque concettualizzazione di giustizia ('adl) non può prescindere dalla centralità della volontà divina nello stabilire attraverso 
la "Via" (šarīah) ciò che "giusto" (haqq) per il musulmano credente (Iqbal, 2006:94-95). Come riassume efficacemente Santillana (1926),

[s]e è vero che la comunità musulmana ha per capo Dio stesso, la legge altro non è se non la volontà di Dio, la norma data da Allāh legislatore al Popolo da lui prescelto, e secondo il quale lo giudicherà. [...] Diritto e religione, legge e morale sono i due aspetti di quella stessa volontà per cui è stata fondata e si regge la comunità musulmana; ogni questione di diritto è anche un caso di coscienza, e la giurisprudenza poggia in ultima analisi sulla teologia (5).

Proprio dalla teologia ulteriori elementi possono contribuire alla comprensione della giustizia economica nell'Islam, che è in primo luogo "sottomissione a Iddio", assoggettamento del credente (muslim) complementare all'assoluta onnipotenza del Creatore. Allāh non è solo "sovrano" di tutto l'universo, ma anche unico "attore" del creato, laddove all'uomo viene attribuito il ruolo di "agente" performante la volontà divina (Watt, 1985): in altri termini, l'apparente molteplicità del creato riflette solo imperfettamente l'unità (tawhīd) della Verità, come rivelata dalla šarīah.

In questa costante tensione tra onnipotenza/sottomissione espressa dall'inscindibile legame "attore"/“agente", è il "precetto" divino (hukm) ad acquisire un ruolo chiave. Come riporta nuovamente Santillana (1926), citando al-Ġazālī, "[l]a legge, hukm, è la parola diretta da Allāh (hiṭāo Allāh) (s'intende mediante il suo Profeta) e che si riferisce agli atti di coloro che sono tenuti (mukallaf) ad osservare i precetti" (p. 5); corrispondentemente al-Ġazālī spiega che "il precetto $(h u k m)$... denota il dictum della rivelazione in quanto connesso agli atti del mukallaf" (citato da Moosa, 1998:9).

In questa realtà profondamente eticizzata, il precetto $(\mathrm{hukm})$ implica quindi un giudizio divino pre-definito sullo stato ontologico dell'azione, che si manifesta palesemente nella nota tassonomia della "quintuplice qualificazione" (al-ahkām al-ḩamsah), laddove il giudizio etico dell'azione non è "costituito" dall'uomo, ma già "collocato" in uno dei cinque status pre-definiti da Dio (Stelzer, 2008:165). Al tempo stesso, nella concezione islamica di giustizia, il precetto divino $(\mathrm{hukm})$ si lega saldamente alla nozione di "diritto" come di "obbligazione", riassunti entrambi nell'idea di haqq (Kamali, 1993:357). Mentre infatti il precetto definisce lo stato etico dell'azione, i "diritti” personali (ḥuqūq) sono il "mezzo" attraverso cui il Creatore realizza (nel senso proprio di rendere reale nel creato) tale stato etico. In questo modo le nozioni di "realtà", "verità" e "dovere" si intrecciano tra loro, fino a promuovere 
a vision of reality as being in its essence imperative, a structure not of objects but of wills. The moral and ontological change places, at least from our point of view. It is the moral, where we see the 'ought', which is a thing of descriptions, the ontological, for us the home of the 'is', which is one of demands. [...] The 'real' here is a deeply moralized, active, demanding real, not a neutral, metaphysical 'being', merely sitting there awaiting observation and reflection; a real of prophets not philosophers.

GEERTZ, 1983:187-188

Questa premessa relativa alla concezione etico-giuridica della realtà come creazione di Iddio (nell'ottica della giustizia legale) consente a questo punto una migliore comprensione della natura del diritto personale (haqq) nell'Islam, e della sua distanza concettuale dalla tradizione giuridica occidentale.

Mentre infatti in quest'ultima tanto la definizione quanto l'attribuzione dei diritti sono intese come "poteri attribuiti alla persona" (Chehata, 1973:179), nell'Islam l'haqq si inserisce pienamente in una concezione etica del reale come prodotto della volontà divina, acquisendo così una connotazione di tipo "tangibile", "reale" ("realtà" è appunto uno dei vari significati di haqqq, come "verità" o "diritto": Lane, 1865), e diventando una sorta di entità "concreta" (segno palese della creazione divina) la cui giusta "collocazione" (ossia, attribuzione al singolo) è già stabilita da Allāh, in un'ottica non di contrapposizione o competizione tra individui, ma di cooperazione e condivisione nell'unico disegno divino. Coerentemente, ben distanti dall'idea di competizione tra soggetti economici che è inerente al capitalismo di stampo occidentale, i diritti patrimoniali non sono intesi come "porzioni" separate di giustizia da "dividere", in modo da essere allocati a livello distributivo sui singoli, ma come "partecipazione" dell'unica giustizia già predisposta da Dio. Così, il concetto di ḥaqq non può essere concepito in termine di un "diritto" del singolo in contrapposizione al "diritto" altrui, ma sia in termini di "diritto" che di "obbligazione": un "diritto" che acquista senso solo nell'unità di due elementi in equilibrio, come riflesso dell'unità della creazione (tawhīd) cui l'uomo partecipa in qualità di agente della volontà divina.

Ecco allora che a partire da una diversa antropologia giuridica relativa ai canoni della giustizia legale, l'Islam giunge ad elaborare un'autonoma concezione della giustizia economica in cui il principio universale del suum cuique tribuere ("dare a ciascuno quanto gli è dovuto") si declina nel senso di collocare il "reale" (i.e. il "diritto", haqq) nel luogo (i.e. "presso l'individuo") cui è attribuito secondo giustizia ('adl): taqrïr al-ḩaqq makānahu huwa al-'adl, nelle parole di Ḥamīd al-Dīn al-Kirmānī, uno dei più importanti pensatori e teologi del 
periodo fatimide (XI secolo). Proprio su questo detto si è soffermato Smirnov (1996), la cui indagine comparativa tra pensiero economico occidentale e musulmano è particolarmente funzionale alla nostra discussione, richiamando la metafora della "bilancia" per chiarire ulteriormente come la "realtà" dell'haqq porti ad una teoria economica volta non tanto alla "divisione", quanto all'equa "partecipazione" alle risorse.

Nell'Islam, infatti,

a right is a sort of substance that has constant volume, of which some parts may happen to be not where they belong, not in the due place: and justice means the necessity of returning them to where they should be. It is not at all by chance that the definition of justice already cited mentions 'establishing the rights (haqq) in its [due] place: each of the rights due has its own 'place' (makān).

SMIRNOV, 1996:344

Alluding to the archetype of the scales, one may say that Western thinking is concerned with the pans of the scales and their contents, while for classical Islamic thought the stress lies on the central balancing pivot. It is making one equal to the other (equality between two necessarily separate entities) that is important in the first case, and theoretical discussion tries to determine the accuracy of this equalizing ... In the second case it is the fact of balancing the opposites that is important, this balance being reached by means of the centering and mediating pivot; the theoretical task is to find out how the two might be linked to form a balanced unity and what the conditions are for such a linkage.

IBIDEM:346-347

In che modo quindi tale concettualizzazione del diritto come "una sostanza dal volume costante", le cui parti vanno collocate "in capo al soggetto cui sono attribuite secondo giustizia" incide ulteriormente a livello di giustizia distributiva e commutativa? E come una razionalità economica centrata sul "perno di equilibrio della bilancia" (Islam) si distingue da una razionalità avente per riferimento, viceversa, i "piatti della bilancia ed i loro contenuti" (Occidente)?

In estrema sintesi, si può asserire che mentre il pensiero economico occidentale si declina in termini di un'equa divisione delle risorse disponibili, la razionalità economica islamica, a partire dal presupposto dell'unicità della giustizia e della creazione divina (tawhìd), si indirizza invece verso un'equa partecipazione alle risorse, concepite, come già accennato, non tanto in 
termini di "porzioni" di giustizia contrapposte ed in competizione tra loro, ma come entità mutualmente corrispondenti. Dai piatti (separati) della metaforica bilancia si passa appunto al perno centrale di equilibrio, e tutti gli sforzi concettuali non sono tanto volti a "rettificare" uno sbilanciamento eventualmente esistente, quanto a "mantenere" l'unità della giustizia voluta da Allāh (Cattelan, 2013a).

Ma la concezione "tangibile" dell'haqq spinge anche oltre il livello di divergenza concettuale tra pensiero economico occidentale e musulmano, fino a suffragare appunto l'asserzione di un paradigma scientifico alternativo.

Se l'haqq non è altro che il "realizzarsi" del precetto $(h u k m)$ che si fa "creato" nel diritto del singolo al momento dell'adempimento della volontà divina, assistiamo infatti ad una concettualizzazione della giustizia economica in cui "ciò che si vuole non è assicurare la libertà del soggetto giuridico, quanto garantire qualcosa di reale alla persona" (Smirnov, 1996:344). Ancora una volta i parametri del pensiero economico occidentale vengono lasciati a margine: lo scambio di beni non è tanto espressione di libertà commerciale (caposaldo del capitalismo occidentale), quanto garanzia che tale libertà sia espressione della "verità" (haqq) del Verbo che si realizza, concretizzando il "diritto" soggettivo (nuovamente, haqq) nell'attribuzione di un bene reale all'individuo che è agente di Dio (muslim, appunto).

In definitiva, l'insieme dei presupposti sopra delineati conducono l'economia islamica ad un paradigma scientifico effettivamente non riconducibile, a mio giudizio, a quello del pensiero occidentale, proponendo un modello alternativo di crescita che può essere riassunto in tre componenti fondamentali:

1) il primato dell'economia reale su quella finanziaria (come espressione di una giustizia legale incentrata sul concretarsi dell'hukm nell'haqq);

2) l'esigenza di un equilibrio costante nelle transazioni commerciali (la giustizia commutativa dei divieti di ribā, ġarar e maysir);

3) una gestione condivisa del rischio, di stampo equity-based e necessariamente asset-backed, in sostituzione dell'approccio debt-based proprio del capitalismo convenzionale (come espressione di una giustizia distributiva fondata su canoni di partecipazione e cooperazione nell'economia reale, e volta ad un profitto condiviso).

Ognuna di queste componenti trova la propria giustificazione etico-razionale nelle premesse culturali fin qui illustrate, come espressione appunto di un modello economico alternativo a quello occidentale (Cattelan, 2013b), che è attualmente quello cui si ispira il sistema finanziario islamico (vedi § 1). 
Così, nell'ambito dell'etica islamica (1) il primato dell'economia reale sulla finanza è logica conseguenza dell'haqq come attribuzione di un "qualcosa di reale alla persona", secondo un'ottica di giustizia legale culturalmente orientata.

In riferimento alla giustizia commutativa, attinente non tanto all'attribuzione dei diritti patrimoniali al singolo, ma ai rapporti commerciali dei singoli tra loro, è invece (2) l'esigenza di un equilibrio tra le contro-prestazioni nel sinallagma a contraddistinguere l'attività di commercio, così come concettualizzato nei divieti di ribā, garar e maysir. Al riguardo, come dimostrato da Saleh (1992) ed El-Gamal (2000 e 2006), il divieto di ribā non riguarda solo l'interesse, ma ogni forma di ingiustizia quantitativa nello scambio, così come le proibizioni di garar (incertezza) e maysir (speculazione) declinano tale giustizia commutativa in termini di trasparenza della transazione. Ancora una volta l'immagine della "bilancia" illustra tale concetto nel mantenimento di una relazione simmetrica tra le controparti, rispetto alle quali la volontà divina concretizzata nell'haqq si fa perno centrale nell'unità concettuale delle reciproche posizioni (Cattelan, 2009).

Questa razionalità economica si completa infine attraverso un modello di crescita basata su una (3) preferenza per investimenti che siano connessi a beni concreti (asset-backed) ed alla partecipazione nel rischio di impresa (equity-based), con distribuzione condivisa dei profitti, come espressione di cooperazione nell'economia reale e con una corrispondente marginalizzazione sia di prodotti finanziari di debito (come le obbligazioni, basate sulla percezione di un interesse e quindi produttive di $r i b \bar{a}$ ) che di natura aleatoria o speculativa (e quindi invalidi per la presenza di garar e maysir) (proponendo così un'antropologia del rischio economico specifica all'Islam: in tal senso, Cattelan, 2014).

In conclusione, superando l'iniziale (pre-)giudizio culturale sull'economia islamica come prodotto di matrice puramente politico-ideologica, incapace a mio avviso di riconoscerne correttamente i presupposti etico-razionali, proprio la reciproca interazione tra i cardini di (1) economia reale, (2) equilibrio transattivo e (3) partecipazione condivisa al profitto/rischio di impresa può essere assunta a sostegno della tesi della sussistenza di un paradigma scientifico autonomo rispetto a quello occidentale.

Altro problema, tuttavia, è individuare come questo paradigma teorico possa farsi effettivamente promotore di un capitalismo alternativo nella pratica del commercio internazionale, e se questo pluralismo economico richieda una rinnovata percezione dei rapporti Occidente/Islam. Su queste questioni saranno avanzate alcune brevi riflessioni conclusive nel paragrafo successivo. 
Partendo dall'attualità politica del mondo musulmano e dalla recente crescita del mercato finanziario islamico a livello globale, questo breve saggio ha voluto esaminare sinteticamente i presupposti concettuali, i contenuti e le finalità dell'economia islamica come paradigma autonomo rispetto al capitalismo convenzionale.

Se infatti l'economia islamica può essere contraddistinta (in chiave eticoreligiosa) alla luce dell'organizzazione delle relazioni di mercato in conformità alla šarīah, è da un punto di vista logico-concettuale che queste sue specifiche "radici" (ușül) delineano una "bilancia" di giustizia sociale che (in chiave etico-razionale) si ramifica (nel senso di alimentare i "rami", furü, della vita morale del credente musulmano) verso parametri di (1) economia reale; (2) equilibrio transattivo; (3) partecipazione condivisa al profitto/rischio di impresa.

Come sarà apparso al cultore di diritto islamico, l'immagine precedente, in cui la metafora della "bilancia" incontra quella dello sviluppo delle "radici" $(u s ̧ \bar{u} l)$ in "rami" ( furū $)$, richiama il paradigma della giurisprudenza musulmana (figh), laddove il corpus normativo (i "rami" del diritto, furü 'al-figh) viene concepito come prodotto dei fondamenti della rivelazione divina e della sua interpretazione ermeneutica (le "radici" del diritto, ușül al-figh). Tale riferimento non è casuale, sia nell'ottica dell'unità delle scienze islamiche secondo i canoni dell'epistemologia musulmana (per cui anche l'economia islamica, come il diritto, è frutto della rivelazione di Allāh), sia nella prospettiva di evidenziare, da un punto di vista inter-disciplinare, come tanto nel figh quanto nell'economia islamica si possa riconoscere una specifica razionalità (in senso dogmatico) a partire da fondamenti morali-religiosi.

Concludendo, esiste allora una "via islamica al capitalismo", fautrice di un paradigma (Kuhn, 1970) alternativo a quello occidentale?

Mentre il "naturale" connubio tra fede e mercato è stato spesso più addotto che investigato in una letteratura che riduce l'economia islamica a "mitologia" (Declich, 2012, in critica ad Atzori, 2010), il presente elaborato ha cercato di sottolineare come sia invece possibile interpretare i presupposti etico-religiosi dell'Islam come origine di una razionalità economica alternativa a quella del pensiero occidentale, e fautrice di un paradigma autonomo, secondo cardini di (1) economia reale, (2) equilibrio transattivo e (3) partecipazione condivisa al profitto/rischio di impresa.

Risolto positivamente il quesito sopra posto in chiave teorica, ci si può chiedere fino a che punto questo paradigma possa effettivamente farsi promotore di un diverso capitalismo (Çizakça, 2011), in chiave più propriamente pratica. 
La profonda crisi finanziaria che ha investito il capitalismo convenzionale a partire dal 2008 sta certamente portando a rivalutare in senso critico il modello di crescita da esso promosso, con un'evoluzione degli scambi commerciali che sperimenta oggi nuovi modelli di interazione economico-sociale (si pensi in primo luogo, per esempio, al fiorente mercato della c.d. sharingeconomy). Così, le critiche mosse all'eccessiva finanziarizzazione dell'economia di mercato sembrerebbero accogliere parametri di favore rispetto all'economia reale, che appartengono di certo anche all'Islam.

In questo senso, la disamina dell'economia islamica condotta in queste pagine, alla luce delle sue peculiarità tanto valoriali quanto razionali, può farsi strumento non solo di un più forte dialogo interculturale tra l'Occidente $\mathrm{e}$ l'Islam, ma anche di una nuova via verso un capitalismo "plurale", capace di "aprire" il mercato a teorie alternative di giustizia economica in grado di coesistere ed influenzarsi reciprocamente (Cattelan, 2013a). Proprio questo pluralismo, nel contesto di un più ampio discorso su economia, lavoro e società, rappresenta a mio giudizio uno dei possibili portati di un nuovo approccio al mondo musulmano, capace di condurre al superamento di stereotipi di matrice post-coloniale/orientalista nella "narrazione" dei rapporti Occidente/Islam (Dabashi, 2012), cui lo studio critico dell'economia islamica può certamente contribuire.

\section{Bibliografia}

AA.vv. Islam e finanza. Religione musulmana e sistema bancario nel Sud-est asiatico. Torino, Edizioni della Fondazione Giovanni Agnelli, 1988.

AFMI (African Financial Market Initiative). "Tunisia to delay sukuk issue until third quarter of 2015". Articolo disponibile online sul sito http://www.africanbondmarket .org (ultimo accesso 15 settembre 2015).

Aliboni, R. (ed.). Banca e finanza islamica: autonomia e cooperazione. Roma, Camera di Commercio Italo-Araba, 2003.

Al-Makarim, Z.A. Ilm al-'adl al-iqtișādī. Il Cairo, Dār al-turāt, 1974.

Alvaro, S. La finanza islamica nel contesto giuridico ed economico italiano (Quaderni Giuridici n. 6). Roma, СоNsoв, Commissione Nazionale per la Società e la Borsa, 2014.

Askari, H.; Taghavi, R. “I fondamenti di principio di un'economia islamica”. Moneta e Credito, LVIII, 232 (2005), p. 175-194.

Atzori, D. Fede e mercato: verso una via islamica al capitalismo?. Bologna, il Mulino, 2010. 
Ayub, M.. Understanding Islamic Finance. Chichester, John Wiley \& Sons Ltd., 2007.

Ballardini, B. IsIS . Il marketing dell'apocalisse. Milano, Baldini \& Castoldi, 2015.

Bausani, A. Il Corano (trad.). Milano, Rizzoli, 1988.

Biancone, P.P. Il bilancio della banca islamica e la rappresentazione dei principali contrattifinanziari. Milano, FrancoAngeli, 2012.

Brunschvig, R. "Conceptions monétaires chez les juristes musulmans (VIII-XVII siècles)". Arabica, 14, 2 (1967), p. 113-143.

Cattelan, V. "From the concept of haqq to the prohibitions of ribā, gharar and maysir in Islamic finance". International Journal of Monetary Economics and Finance, 2, 3-4 (2009), p. 384-397.

Cattelan, V. "Islamic finance and ethical investments. Some points of reconsideration". In: Khan, F.M.; Porzio, M. (eds). Islamic Banking and Finance in the European Union. A challenge. Cheltenham e Northampton, Edward Elgar Publishing, 2010, p. 76-87.

Cattelan, V. (ed.). Islamic Finance in Europe: Towards a Plural Financial System. Cheltenham and Northampton, Edward Elgar Publishing, 2013a.

Cattelan, V. "A glimpse through the veil of Maya: Islamic finance and its truths on property rights". In: Cattelan, V. (ed). Islamic Finance in Europe: Towards a Plural Financial System. Cheltenham e Northampton, Edward Elgar Publishing, 2013b, p. 32-51.

Cattelan, V. "Shari'ah economics as autonomous paradigm: theoretical approach and operative outcomes". Journal of Islamic Perspective on Science, Technology and Society, 1, 1 (2013c), p. 3-11 (disponibile online sul sito http://www.jistecs.org).

Cattelan, V. "In the Name of God: managing risk in Islamic finance". EABH Working Papers Series, no. 7 (2014), disponibile online all'indirizzo www.eabh.info/publications/eabh-papers, p. 1-14 (ultimo accesso 15 settembre 2015).

Chapra, M.U. Obiettivi dell'ordine economico islamico (tr. P. Visani). Carmagnola, Arktos, 1979.

Chapra, M.U. The Future of Economics: an Islamic Perspective. Leicester, The Islamic Foundation, 2000.

Chehata, C. Études de droit musulman. 2. La notion de responsabilité contractuelle. Le concept de propriété. Paris, Presses Universitaires de France, 1973.

Çizakça, M. Islamic capitalism and finance. Origins, evolution and the future, Cheltenham e Northampton, Edward Elgar Publishing, 2011.

Dabashi, H. The Arab Spring: the End of Post-colonialism. London and New York, Zed Books, 2012.

Declich, L. "Il mito dell'economia islamica". In: Tutto in 30 Secondi. Appunti e Note sul Mondo Islamico Contemporaneo, 2012, articolo disponibile online sul sito in3osecondi.altervista.org (ultimo accesso 28 settembre 2015).

Dell'Atti, A.; Miglietta, F. (eds). Fondi sovrani arabi e finanza islamica. Milano, EGEA, 2009 . 
El-Gamal, M.A. "An economic explication of the prohibition of ribā in classical Islamic jurisprudence". In: Proceedings of the Third Harvard University Forum on Islamic Finance. Center for Middle Eastern Studies. Cambridge, MA, Harvard University Press, 2000.

El-Gamal, M.A. Islamic finance. Law, economics, and practice, New York, Cambridge University Press, 2006.

Ercanbrack, J. The transformation of Islamic law in global financial markets. Cambridge, Cambridge University Press, 2015.

Francesca, E. Economia, religione e morale nell'Islam. Roma, Carocci, 2013.

Geertz, C. Local knowledge. Further Essays in Interpretive Anthropology. New York, Basic Books, 1983.

Gimaret, D. Théories de l'acte humain en théologie musulmane. Paris-Leuven, VrinPeeters, 1980.

Gimaret, D. La doctrine d'al-Ash'arī, Paris, Cerf, 1990.

Gimigliano, G.; Rotondo, G. (eds). La banca islamica e la disciplina bancaria europea. Atti del Convegno Internazionale di Studi, Napoli, 8-9 aprile 2005. Milano, Giuffrè, 2006.

Giustiniani, E., Elementi difinanza islamica. Torino, Rist, 2006.

Gomel, G. (ed.). Finanza islamica e sistemi finanziari convenzionali. Tendenze di mercato, profili di supervisione e implicazioni per le attività di banca centrale (Questioni di Economia e Finanza, Occasional Paper n. 73). Roma, Banca d'Italia, 2010.

Guandalini, M.; Uckmar, V. Gulf\&Med. Il mercato, gli investimenti e la finanza islamica. Milano, Mondadori, 2015.

Hamaui, R.; Mauri, M. Economia e finanza islamica. Bologna, il Mulino, 2009.

Hasanuzzaman, S.M. "Definition of Islamic economics". Journal for Research in Islamic Economics, 1, 2 (1984), p. 51-53.

Iqbal, Z. "Contemplating an Islamic theory of justice: situating tradition amidst modernity”. Review of Islamic Economics, 10, 1 (2006), p. 91-121.

Islahi, A.A. History of Economic Thought in Islam: a Bibliography. Jeddah, Islamic Economics Research Centre, King Abdulaziz University, 2007.

Ismail, I.M. L'Islam e le teorie economiche odierne (tr. P. Visani). Carmagnola, Arktos, 1980.

Kamali, M.H. "Fundamental rights of the individual: an analysis of haqq (right) in Islamic law". The American Journal of Islamic Social Sciences, 10, 3 (1993), p. 340-366.

Khan, A. "Islamic economics, nature and need". Journal for Research in Islamic Economics, 1, 2 (1984), p. 55-61.

Khan, M.F.; Porzio, M. (eds). Islamic Banking and Finance in the European Union. A Challenge. Cheltenham e Northampton, Edward Elgar Publishing, 2010. 
Kuhn, T.S. The structure of scientific revolutions. Chicago, University of Chicago, $1970^{2}$.

Kuran, T. "The economic system in contemporary Islamic thought: interpretation and assessment". International Journal of Middle East Studies, 18, 2 (1986), p. 135-164.

Lane, E.W. Arabic-English Lexicon. 8 vol., 1865 (-1893).

Le Goff, J. La bourse et la vie. Économie et religion au Moyen Age. Paris, Hachette, 1986.

Le Goff, J. Le Moyen Age et l'argent. Essai d'anthropologie historique. Paris, Perrin, 2010.

Lofty, A. "Egypt Islamists draft code to boost Islamic banks". Articolo disponibile online sul sito http://www.reuters.com (ultimo accesso 15 dicembre 2016). Reuters, 11 giugno 2012.

Lofty, A.; Vizcaino, B. "Political divisions slow Islamic finance in Egypt". Articolo disponibile online sul sito http://www.reuters.com (ultimo accesso 18 dicembre 2016). Reuters, 22 aprile 2012.

Malik, A.; Awadallah, B. The Economics of the Arab Spring. CsAE Working Paper WPS/2011-23, Centre for the Study of African Economics, Oxford, University of Oxford, 2011.

Moosa, E. "Allegory of the rule (hukm): law as simulacrum in Islam?". History of Religions, 38, 1 (1998), p. 1-24.

Nelson, B. The Idea of Usury. From Tribal Brotherhood to Universal Otherhood. Princeton, NJ, Princeton University Press, 1949.

Philipp, T. "The idea of Islamic economics”. Die Welt des Islams, Nr. 1/4 (1990), p. 117-139.

Piccinelli, G.M. Banche islamiche in contesto non islamico: materiali e strumenti giuridici. Roma, Ipocan, 1996.

Porzio, C. (ed.). Banca e finanza islamica. Contratti, peculiarità gestionali, prospettive di crescita in Italia. Carefin, Centre for Applied Research in Finance, Roma, Bancaria, 2009.

Ray, N.D. "The medieval Islamic system of credit and banking: legal and historical considerations". Arab Law Quarterly, 12, 1 (1997), p. 43-9o.

Rodinson, M. Islam et capitalisme. Paris, Éditions du Seuil, 1966.

Roy, O. "Europe and the Mediterranean: when the obsession for security misses the real world”. EUI Working Papers RSCAS 2012/20, Robert Schuman Centre for Advanced Studies, Firenze, Istituto Universitario Europeo, 2012.

Russo, V. I contratti sharĩa compliant. Valori religiosi e meritevolezza degli interessi. Napoli, Edizioni Scientifiche Italiane, 2014.

Saleh, N. Unlawful Gain and Legitimate Profit in Islamic Law. Ribā, gharar and Islamic Banking. London, Graham \& Trotman, 1992.

Santillana, D. Istituzioni di diritto musulmano malichita con riguardo anche al sistema sciafita, Vol. I. Roma, Istituto per l'Oriente, A.R.E. 1926.

Scarcia Amoretti, B. "Il concetto di libertà nei testi di economia islamica: alcune osservazioni”. Alifba, $6 / 7$ (1986), p. 47-54, Palermo, Centro Culturale Al-Farabi. 
Scarcia Amoretti, B. Profilo dell'economia islamica. Palermo, Centro Culturale AlFarabi, 1988.

Serafini, M. "Isis avvia il conio del dinaro d'oro: « Distruggeremo il capitalismo »". Corriere della Sera, 31 agosto 2015 .

Smirnov, A. "Understanding justice in an Islamic context: some points of contrast with Western theories". Philosophy East and West, 46, 3 (1996), p. 337-350.

Solovieva, D.; Wahba, A.L. "Egypt signals sukuk intent while preparing foreign bond comeback". Articolo disponibile online sul sito http://www.bloomberg.com (ultimo accesso 18 dicembre 2016). Bloomberg, 29 marzo 2015.

Stelzer, S.A.J. "Ethics". In Winter, T. (ed.). The Cambridge companion to Classical Islamic Theology. Cambridge, Cambridge University Press, 2008, p. 161-179.

TheBanker. Top Islamic Financial Institutions, Special Report. London, Financial Times Ltd., 2013.

Tripp, C. Islam and the Moral Economy: the Challenge of Capitalism. Cambridge, Cambridge University Press, 2006.

Udovitch, A.L. Partnership and Profit in Medieval Islam. Princeton, NJ, Princeton University Press, 1970.

Vadalà, E. Capire l'economia islamica. Patti, Yorick, 2004.

Vizcaino, B. "Tunisia sets up Islamic finance working group". Articolo disponibile online sul sito http://www.reuters.com (ultimo accesso 15 dicembre 2016). Reuters, 15 marzo 2012.

Vogel, E.F.; Hayes, S.L. Islamic Law and Finance. Religion, Risk and Return. The Hague, Kluwer Law International, 1998.

Warde, I. Islamic Finance in the Global Economy. Edinburgh, Edinburgh University Press, 2000.

Watt, W.M. "Islamic alternatives to the concept of free will". In: La notion de liberté au Moyen Age. Islam, Byzance, Occident. Parigi, Les Belles Lettres, 1985, p. 15-24.

Weber, M. The Protestant Ethic and the Spirit of Capitalism. New York, MacMillan, 1958 (orig. Die protestantische Ethik und der Geist des Kapitalismus, 1904-1905). 